\title{
Reconfigurable Architecture Using High Speed FPGA
}

\author{
L. Kessal, R. Bourguiba, D. Demigny, N. Boudouani, M. Karabernou \\ ETIS - UPRESA CNRS 8051ENSEA - Cergy Pontoise University6 Av. du Ponceau, 95014 \\ Cergy Pontoise Cedex, France E-mail: kessal@ensea.fr
}

Abstract: Here, we discuss about Dynamic Reconfiguration (or Run Time Reconfiguration), a technique based on the reuse of the same device (an FPGA configured on the fly) by scheduling the execution of different algorithms building an application. Our project joins the efforts of ten laboratories working on methods and tools for Adequation Algorithm Architecture. The design of a hardware template with such a concept, will help the emergence of new methods for applications development and the benefits estimation of this approach. In this paper, we present our architecture, ARDOISE, dedicated to real time image processing. Then an analytical model is defined in order to compute the limits and the performances expected in the use of the dynamic reconfiguration scheme.

Key words: dynamic reconfiguration, run time reconfiguration, image processing, fpga

\section{INTRODUCTION}

Ten research French teams study and build a hardware architecture (ARDOISE) dedicated to real time image processing. This architecture uses fast or dynamic reconfiguration provided in new FPGA circuits. This new kind of devices can be totally or partially configured in less than $1 \mathrm{~ms}$ and 
their processing speed is approximately $1 / 3$ of ASIC speed. The basic idea is to chain algorithms used in image segmentation on the same hardware structure, by reconfiguring few devices, several times during the processing of a single image. This is equivalent to assigning the same hardware resource: an FPGA device to execute a sequence of algorithms according to a defined scheduling. Using this concept, the final system allows the implementation of applications on FPGA as fast as on ASIC circuits. However, it authorizes a same flexibility level, formerly, reserved to microprocessors. On the other hand, this project will help in estimating the contribution of the Dynamic Reconfiguration (DR) paradigm to the performances of hard wired systems.

In this paper, we briefly present the ARDOISE architecture, which can be reconfigured entirely or partially. Next, we discuss (section 3) the performances limits of a Dynamically Reconfigured Architecture (DRA) and give an evaluation of the silicon area gain expected, compared to a classical solution based on a set of statically configured FPGA circuits. In the five section, we compare the performances of three possible implementations of a DRA:

1. one large FPGA is reconfigured at run time,

2. two FPGA are alternatively reconfigured at run time, in order to mask the configuration delays,

3. two FPGA are reconfigured at run time, in parallel.
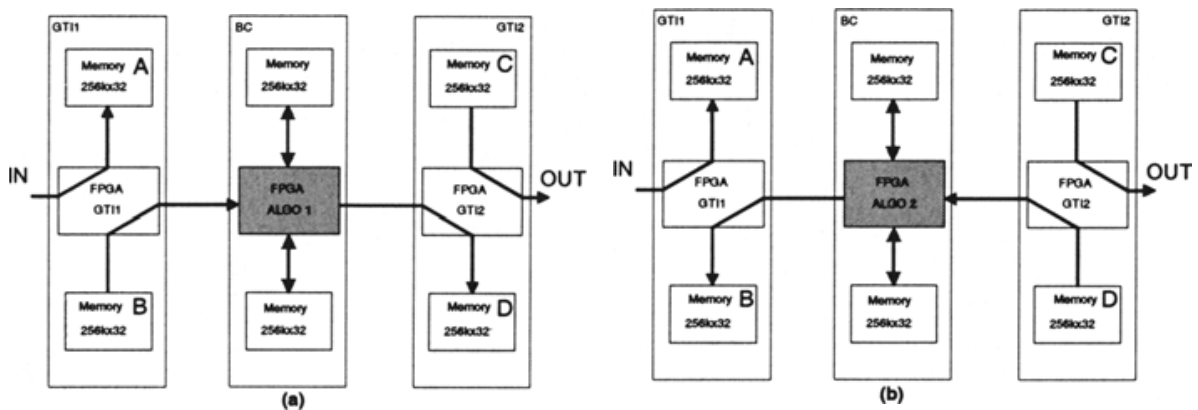

Figure 1. Processing two algorithms on ARDOISE architecture

\section{THE ARDOISE ARCHITECTURE}

The ARDOISE architecture [1,2] is based on three identical modules (figure 1). Each one encloses an FPGA, with a $45 \mathrm{~K}$ gates count connected to two local memories to store intermediate results. 
The GTI1 and GTI2 modules provide an interface with the input and output systems, that allows desynchronization between the central computing module, $\mathrm{BC}$, and the video acquisition system which occurs often at lower frequency than the maximum FPGA abilities. The different treatments are swapped in the central module. Partial results will be stored in the local memories with different memory models, while computing or reconfiguring.

\section{PERFORMANCES AND LIMITS OF DRA}

The main objective of Dynamic Reconfiguration is to allow a system to react during run time and choose the right algorithm at the right time offering performances close to those of the hard-wired systems. Compared to a static solution, DR does not improve the execution speed of an application. However, it reduces and optimizes the use of the silicon area in the FPGA. Bertin suggested [3] the definition of the power $P_{u}$ needed by an application in the case of the static architecture as the product of the number of gates $G_{s}$ required and of the computing frequency. In the same way, the power of an architecture can be defined as the product of the number of equivalent gates of the architecture and the maximum frequency $F_{t}$ that can be used. The application which uses $\mathrm{G}_{\mathrm{s}}$ gates in a static architecture, is splitted in $C$ parts and then computed on an image with $\mathrm{C}$ configurations of the FPGA. We have already shown $[1,2]$ that there is a limit of the useful application power when using DR:

$$
P_{\text {dynamic }}=G_{d} F_{t}\left(1-C \frac{G_{d}}{V_{c} T}\right)
$$

$\mathrm{G}_{\mathrm{d}}$ represents equivalent gate count of the dynamically reconfigurable architecture and $\mathrm{T}$ the computing time of a block of data. For an image rate of 25 images $/ \mathrm{s}$, the block duration is $T=40 \mathrm{~ms}$.

The limit depends on the configuration speed $V_{c}$ (expressed in number of gates configured per second) and of the maximal computing frequency $F_{t}$ provided by the technology.

\subsection{Maximal power and complexity limits}

For a given number of configurations, when $G_{d}$ increases, there is a maximum value which can be reached by $P_{u}$ : 


$$
P_{u} \max =\frac{F_{l} G_{d}}{2} \text { with } G_{d}=\frac{V_{c} T}{2 C}
$$

For each configuration $\mathrm{p}, \beta_{p}$ data are processed in parallel (parallelism rate). The maximal complexity of an application implemented with DR is then defined:

$$
G_{s} \max =\frac{V_{c} T}{2 C} \sum_{P=1}^{c} \frac{1}{\beta_{p}} \leq \frac{V c T}{2}
$$

This equation shows that there is a limit in the hardware complexity of an application that can be implemented with DR. This limit is exclusively a function of the configuration speed authorized by the technology.

\subsection{Maximum gate count of a DRA}

If the image size is $\mathrm{N}$ pixels, the sampling frequency of the pixel is:

$$
F_{e}=\frac{N}{T}
$$

For a given application $\left(\mathrm{F}_{\mathrm{e}}, \mathrm{T}, \mathrm{G}_{\mathrm{s}}\right)$, the useful power is:

$$
P_{\text {static }}=G_{s} F_{e} \leq P_{\text {dynamic }}
$$

We obtain:

$$
G_{d} \min =\frac{G_{s} F_{e}}{F_{t}\left(1-C \frac{G_{d}}{V_{c} T}\right)}
$$

The implementation according to the DR concept allows a better silicon area reduction with a parallelism rate of $\beta_{p}=1$. In this part, we show that the silicon gain is better when the data acquisition frequency is low compared to that of the processing frequency. The rate is defined as:

$$
\frac{G_{s}}{G_{d} \min } \approx \frac{F_{t}}{F_{e}}
$$


Performances on silicon reduction appears when dynamic reconfiguration is used at less than $20 \%$ of the limits.

\subsection{Performances}

In order to simplify the expressions and to make the figures analysis easier, we suppose that all configurations use the same data parallelism rate $\beta$. Respecting the real time constraints, the following relation has to be considered:

$$
C\left(\frac{N}{F_{t} \cdot \beta}+\frac{G_{d}}{V_{c}}\right) \leq T .
$$

The following curves, drawn for two different technologies (XILINX 4000 family and ATMEL AT40K family), show the importance of the configuration speed $\mathrm{V}_{\mathrm{c}}$ and of the data parallelism rate $\beta$, on the complexity limit as well as on the substantial silicon area gain.

For example, the speed of the configuration $V_{c}$ of ATMEL AT40K is 100 times faster than XILINX 4000. The complexity of the application should be 100 time greater.

These curves have been drawn with $\mathrm{T}=40 \mathrm{~ms}$ (block duration in image processing).
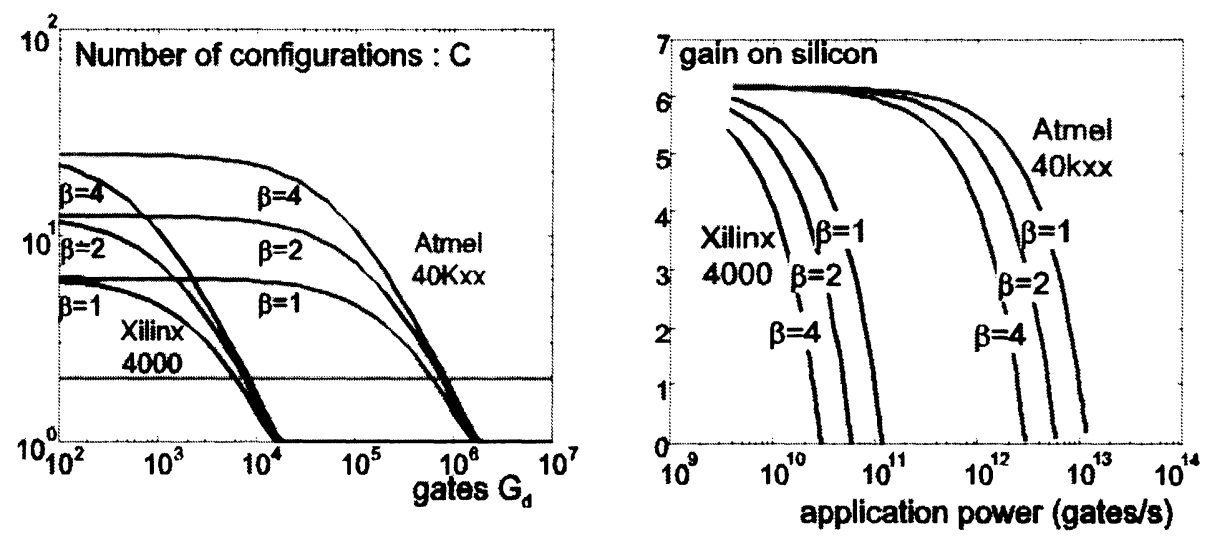

Figure 2. Number of configurations according to the application complexity expressed in terms of gate count and the gain in term of silicon area according to the computing power required by an application 


\section{WHICH TYPE OF FPGA FOR DRA?}

The dynamic reconfiguration is a concept which is not reserved for only FPGA who offer a high configuration speed such as Xilinx XC6200 and Atmel AT40K series. These two FPGA families allow dynamic partial or complete reconfiguration during run-time. This is not currently available in technologies such as the Xilinx 4000 family, because devoid of an interface of fast configuration times. According to the curves shown in $\S 3.3$, built architecture with only one FPGA will have significant reductions in efficiency.

The performances can be degraded by the reconfiguration time of the FPGA hardware. However, this does not mean that the use of classical FPGA circuits is excluded in the design of the DRA. There has been much interest in the development of dynamic reconfiguration architecture using reconfigurable FPGA connected in various topology. These last years, a great number of systems are built by teams of research integrating mechanisms aiming to reduce the effect of the configuration times: configuration data caching, bit-stream compression techniques, masking the configuration, ...

The masking of configuration strategy was studied by $\mathrm{H}$. Guermoud in his thesis [4]. In the following of this article, we compare the performances of this approach with the classical DRA (such as ARDOISE).

\section{DR SYSTEMS WITH OR WITHOUT MASKING ?}

We can make benefit of DR, even with slow configuration speed. One can imagine solutions that use two FPGA, each one with a capacity of $G_{d} / 2$ gates, instead of a single one with $\mathrm{G}_{\mathrm{d}}$ gates large.

Real-time image processing requires complex: Nagao filter, Edges detector, Boundary closing, Regions labeling, ...). The algorithm implementations [5] include a large variety of hardware models (pipeline, data flow, parallelism, ...) and data arrangement. They usually need very high speed memories and data address generator for an effective data organization (data formatting). For example, some operators require to access to many pixels at the same time. That is why, sufficient memory size and high speed access (bandwidth) are very important for image processing. For processing an image, each step requires large frame storage.

ARDOISE architecture module includes 2Mbytes classic SRAM (for random memory access); the memory bandwidth is $400 \mathrm{Mbytes} / \mathrm{s}$.

Now, we calculate and discuss the performances and the silicon reduction of the following solutions: 
1. without masking the reconfiguration time,

2. Masking the reconfiguration time: one device is reconfigured, while the other is computing,

3. Doubling the reconfiguration speed: the two devices are reconfigured at the same time, and then compute together.

The three solutions use the same quantity of hardware resource: total number of gates, memory size and memory bandwidth.

To simplify expressions and figures interpretation, we assume here that the same data parallelism rate $\beta$ is used for each algorithm.

\subsection{Solution without masking}

In this case, a single FPGA with a capacity of $G_{d}$ equivalent gates is used. In order to execute several algorithms, a series of reconfiguration / computation are performed alternatively.

\section{Number of configurations}

$$
C=\frac{T}{\frac{N}{F_{t}}+\frac{G_{d}}{V_{c}}}=\frac{1}{\frac{F_{e}}{F_{t}}+\frac{G_{d}}{V_{c} \cdot T}}
$$

\section{Complexity of the application}

$$
G_{s}=C \cdot G_{d}=\frac{G_{d}}{\frac{F_{e}}{F_{t}}+\frac{G_{d}}{V_{c} \cdot T}}<V_{c} \cdot T
$$

Because dynamic reconfiguration has no sense if $\mathrm{C}<2$, the maximum value of the equivalent gates of the dynamic architecture is:

$$
G_{d} \max =\frac{V_{c} \cdot T}{2}\left(1-2 \cdot \frac{F_{e}}{F_{t}}\right)
$$

The expression of $\mathrm{G}_{\mathrm{s}}$ can spell also:

$$
G_{s}=V_{c} \cdot T\left(1-C \cdot \frac{F_{e}}{F_{t}}\right)
$$


The silicon area reduction $G_{g}$ according to the complexity of the application is:

$$
G_{g}=\frac{G_{s}}{G_{d}}=C=\frac{F_{t}}{F_{e}}\left(1-\frac{G_{s}}{V_{c} . T}\right)
$$

\subsection{DR with masking configuration}

In this case, a series of reconfiguration / computation is applied in alternation to each of the two FPGA (each provided the equivalent of $G_{d} / 2$ gates).

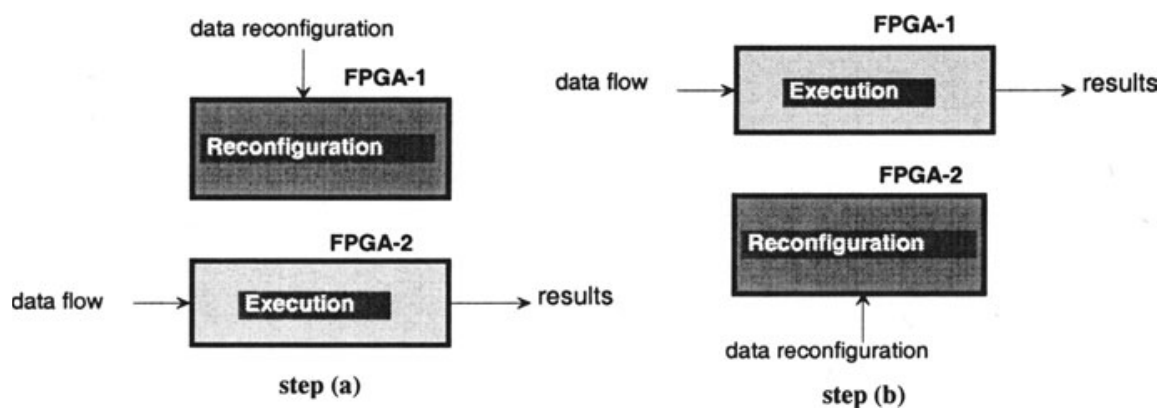

Figure 3. Technique of masking the reconfiguration times

At any step, a phase of reconfiguration of the first FPGA is applied as long as the second FPGA executes a treatment (figure 3a). In the following step, after the reconfiguration time, the first FPGA starts to operate while a configuration is applied to the second one (figure $3 b$ ), and so on...

\section{Number of configurations}

Two possible situations should be considered:

1. In the first situation where the reconfiguration time is lower than the computation, the number of configurations can be written as:

$$
C=\frac{T}{N \cdot \frac{1}{F_{t}}}=\frac{F_{t}}{F_{e}}
$$


2. In the second situation where the time reconfiguration is greater than the computation time, the number of configurations can be expressed as:

$$
C=\frac{V_{c} \cdot T}{\frac{G_{d}}{2}}=\frac{2 \cdot V_{c} \cdot T}{G_{d}}
$$

In this situation the complexity of the application is: $G_{s}=V_{c} T$. In both cases, we can deduce the number of configurations by:

$$
C=\min \left(\frac{F_{e}}{F_{t}}, \frac{2 . V_{c} . T}{G_{d}}\right)
$$

In practice, it's obvious that partial masking of the configuration time is without interest. For this reason, we continue studying only total masking of the configuration time.

\section{Complexity of the application}

$$
G_{s}=C \cdot \frac{G_{d}}{2}=\frac{G_{d} \cdot F_{t}}{2 \cdot F_{e}} \leq V_{c} \cdot T
$$

Dynamic reconfiguration is interesting beyond 4 configurations, the maximum value of the equivalent gates of the dynamic architecture is:

$$
G_{d} \max =2 . V_{c} \cdot T \cdot \frac{F_{e}}{F_{t}} \leq \frac{V_{c} \cdot T}{2}
$$

The silicon reduction $\mathrm{G}_{\mathrm{g}}$ is:

$$
G_{g}=\frac{G_{s}}{G_{d}}=\frac{C}{2}=\frac{F_{t}}{2 . F_{e}}
$$




\subsection{DR with double configuration speed}

Here, the two FPGA devices are reconfigured simultaneously with different configuration data [6]. After the reconfiguration time, each FPGA starts to execute its own correspondent treatment.

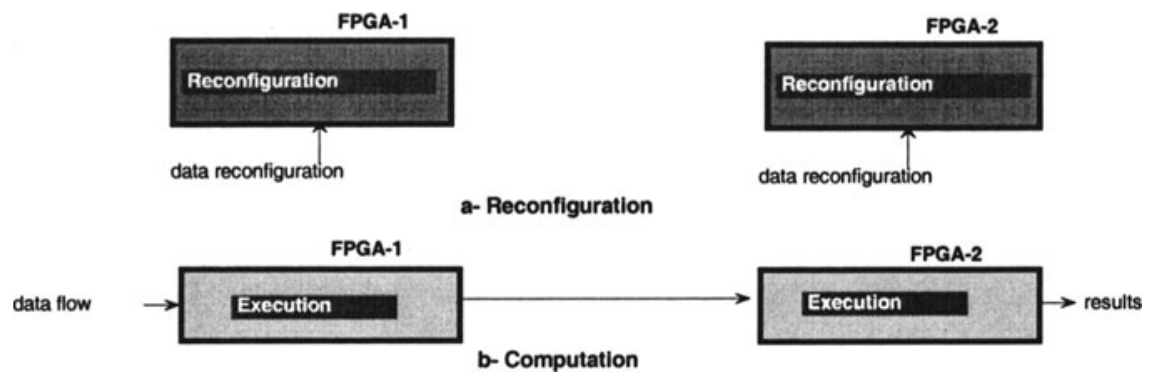

Figure 4. Technique of doubling of the reconfiguration speed

The figure 4 , shows the functionality of this architecture. One is in the situation of a classical dynamic reconfigurable architecture with a double configuration speed. So the complexity is:

$$
G_{g}=\frac{F_{t}}{F_{e}}\left(1-\frac{G_{s}}{2 . V_{c} . T}\right)
$$

The following table summarizes the expression of the silicon area gain for various solutions:

\begin{tabular}{|l|l|}
\hline Normal architecture & $G_{g 1}=\frac{F_{t}}{F_{e}}\left(1-\frac{G_{s}}{V_{c} \cdot T}\right)$ \\
\hline Architecture masking configuration delays & $G_{g 2}=\frac{F_{t}}{2 \cdot F_{e}}$ \\
\hline Architecture with double configuration speed & $G_{g 3}=\frac{F_{t}}{F_{e}}\left(1-\frac{G_{s}}{2 \cdot V_{c} \cdot T}\right)$ \\
\hline
\end{tabular}


The following figure represents the three curves. The silicon gain of the three solutions discussed above are drawn according to the size normalized for a ratio $F_{t} / F_{e}=10$.

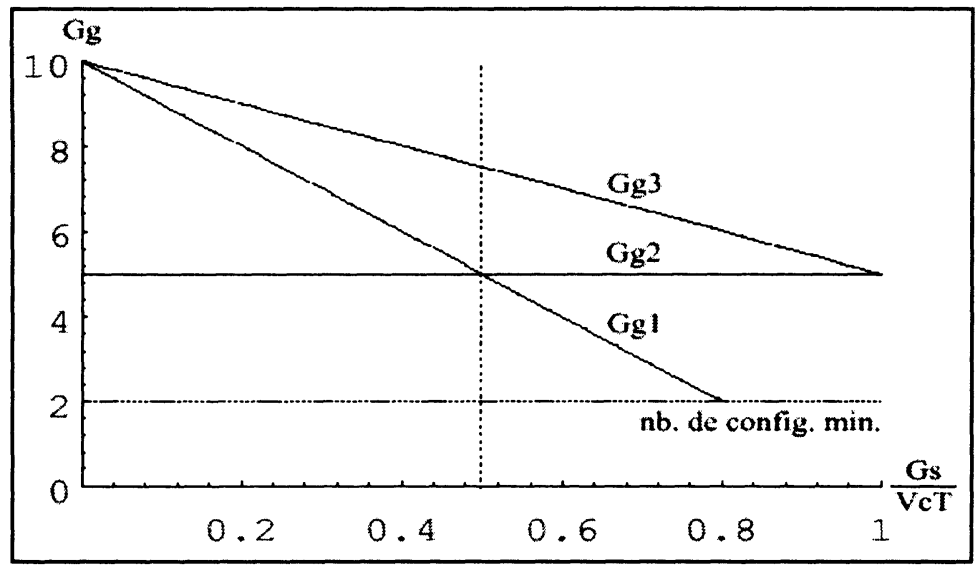

Figure 5. Gain in silicon of the various solutions

Two zones are distinguished. A first zone, in which the masking the of configuration time is advised, the silicon gain of this solution is lower than the classical solution. In the second zone, the masking of configuration time becomes interesting. The dynamic reconfiguration is only interesting, if the reconfiguration time of the FPGA is around of $10 \%$ of the block duration $\mathrm{T}$. That depends on the technology of the FPGA used. It is the case of the circuits AT40K that we use. The technique of masking the configuration becomes interesting if the FPGA has a slower reconfiguration speed. The technique of doubling the speed confirms the importance of the reconfiguration speed. It represents the best solution among the three (less flexible).

This proves that the solution is technological. FPGA circuits should be endowing with mechanisms allowing reconfiguration time reduction (better reconfiguration interface, using configuration data caching, partial reconfiguration, ...). For a given technology, the solution with parallel reconfigurations offers better performances.

The solution based on configuration masking is consequently without great interest. Due to material management in a ping pong manner of the FPGA devices, the system implementation will present some difficulties (complexity in the PCB design). 


\section{CONCLUSION}

In this paper, we presented a new paradigm for designing hardware architecture using dynamically reconfigurable FPGA. We showed the importance of the FPGA reconfiguration time to increase the performances of dynamically reconfigurable systems. We studied and compared the performances with two solutions (masking the reconfiguration delay, doubling reconfiguration speed) using the same hardware resources.

This work indicates that performance increase is possible using doubling reconfiguration speed technique. However, the system hardware realization is more delicate as well as the flexibility is reduced.

The results of this study make our ARDOISE architectural choices are well justified. Indeed, the solution to increase the reconfigurable architecture performances in a significant way consists in enhancement of the FPGA technology: efficient configuration interface, low configuration time, configuration data caching, possibility of partial configuration, ...

Al so, development software tools should contribute to improve reconfigurable architecture performances by including more feature such as configuration data compression, partial and complete configuration management, automatic application partitioning, ...

\section{REFERENCES}

[1] Didier Demigny, Lounis Kessal, Riad Bourguiba and Nassima Boudouani. How to use high speed reconfigurable FPGA for real time image processing ? Proc. International Conf. on Computer Architecture for Machine Perception, pages 240-246, Padova, septembre 2000. IEEE Circuit.

[2] Lounis Kessal, Didier Demigny, Nassima Boudouani and Riad Bourguiba. Reconfigurable hardware for real time image processing. Proc. International Conference on Image Processing, volume 3, pages 159-173, Vancouver, septembre 2000. IEEE ICIP.

[3] P. Bertin, D. Roncin and J. Vuillemin, Programmable active memories: a performance assessment, In F. Meyer auf der Heide, B. Monien, and A. L. Rosenberg, editors, Parallel Architectures and their efficient use, pp. 119-130, Lecture notes in Computer Science, Springer-Verlag, October 1992.

[4] H. Guermoud, Architecure reconfigurables dynamiquement dédiées aux traitements en temps réel des signaux vidéo, Thesis, faculty of Nancy I, France 1997.

[5] Riad Bourguiba, Didier Demigny and Lounis Kessal. Dynamic configuration: a new paradigm applied to real time image analysis. In Proc. The tenth International Conference on Microelectronis, pages 25-28, Monas-tir, Tunisia, December 1998. IEEE Electron Device Society.

[6] Riad Bourguiba. Conception d'architecture matérielles reconfigurables dynamiquement dédiées au traitement d'images temps réel. Thèse de doctorat en sciences spécialité traitement d'images, Université de Cergy Pontoise, juillet 2000. Jury: P. Bertin, D. Demigny, L. Kessal, M. Paidavoine, R. Tourki, S. Weber. 